http://jmscr.igmpublication.org/home/ ISSN (e)-2347-176x ISSN (p) 2455-0450

crossref DOI: https://dx.doi.org/10.18535/jmscr/v9i12.18

Journal Of Medical Science And Clinical Research

\title{
Ocular manifestations of Mucormycosis in patients of COVID-19
}

Authors

\section{Dr Malvika Sharma, Dr Enakshi Bali, Dr Narain Sharma}

\section{Introduction}

Mucormycosis is an angioinvasive disease caused by fungi of the order Mucorales like Rhizopus, Mucor, Rhizomucor, Cunninghamella and Absidi. The prevalence of mucormycosis in India is approximately 0.14 cases per 1000 population, about 80 times the prevalence in developed countries $^{(3)}$. Mucormycosis is a potentially fatal opportunistic infection that can manifest in many different clinical forms, including a rhinocerebral form, in the pulmonary system, central nervous system, gastrointestinal system, and other parts of the body. Rhinocerebral mucormycosis is subdivided into 3 groups: rhinomaxillary, rhinoorbital, and rhino-orbito-cerebral mucormycosis ${ }^{(5)}$. Extensive forms of the disease include ophthalmia and cranial nerve involvement ${ }^{(6)}$. Mucormycosis is more often seen in immunocompromised individuals, and complications of orbital and cerebral involvement are likely in diabetic ketoacidosis and with concomitant use of steroids. The most common risk factor associated with mucormycosis is diabetes mellitus in $\operatorname{India}^{(4)}$. Hence, early diagnosis of this potentially lifethreatening disease and prompt treatment is of prime importance in reducing the mortality rate.

Recently, several cases of mucormycosis in people with COVID-19 have been increasingly reported world-wide, in particular from India. We, hereby, report 4 cases of clinically diagnosed orbital mucormycosis with concurrent COVID-19 illness at our institute over the last 2 months (May and June 2021). All of these patients were admitted as, RT-PCR positive cases of COVID-19 and were being treated for the same in our hospital. Potassium hydroxide $(\mathrm{KOH})$ wet mount and fungal culture/sensitivity were done from biopsy obtained during debridement or from nasal swab obtained during diagnostic nasal endoscopy. Microbiological diagnosis of mucormycosis was proven in three patients. All patients in our series were known diabetics and were receiving intravenous steroids, broad spectrum antibiotics and remedesivir as a part of treatment of COVID19. All patients in our series had received intravenous dexamethasone for COVID-19 disease as per The National Institute of Health recommendations ${ }^{(1)}$. The use of steroids, monoclonal antibodies, and broad-spectrum antibiotics for the management of COVID-19 illness can increase the chances of new-onset fungal infection or exacerbate a preexisting one. ${ }^{(2)}$

\section{Case 1}

72 year old diabetic female patient presented with severe unilateral right sided headache with ipsilateral eye ache for two days, relieved by analgesics. On examination on day 1 , visual acuity was $6 / 12$ in right eye, along with mild ptosis $(<2$ $\mathrm{mm})$, proptosis $(<22 \mathrm{~mm})$, mild periorbital edema, 
and upper lid erythema. There was no restricition of ocular motility in right eye. Visual acuity in left eye was 6/24. No other significant findings except for lenticular changes were seen. CE MRI showed bilateral pansinusitis with presence of multiple T2 hypointense non enhancing area within them likely suggestive of fungal etiology with focal narrow edema in right frontal bone with small subdural component with leptomeninges involvement in right frontal region with peripheral inflammation of right orbit. $\mathrm{KOH}$ smear showed no growth. Diagnostic Nasal Endocopy showed no discoloration but pus discharge in the middle meatus. On Day 3, patient had severe ptosis $(>4 \mathrm{~mm})$, severe proptosis, periorbital edema, conjunctivalchemosis and restricted ocular motility in all gazes. Treatment with Amphotericin B was initiated, and Bilateral FESS was performed.

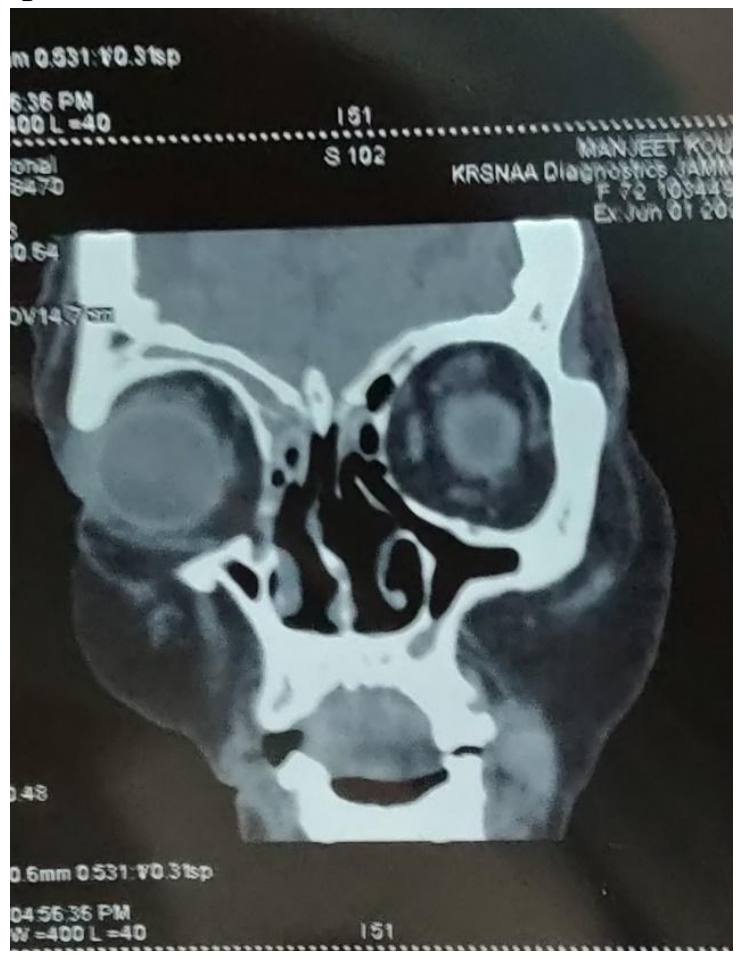

\section{Case 2}

55 year old diabetic male patient presented with painful loss of vision and swelling in left eye with blackish discoloration around left eye. On examination of left eye, visual acuity showed no PL. Patient had severe ptosis, severe proptosis, edematous cornea, conjunctival congestion and chemosis. Anterior chamber had irregular depth.
A characteristic eschar measuring about $2 * 3 \mathrm{~mm}$ was seen at medial canthus extending onto the upper eyelid. There was complete motility restriction in all directions of gaze. CT head and orbit showed mucosal thickening in b/l maxillary, ethmoidal, frontal, and sphenoidal sinuses causing obliteration of osteomeatal unit and frontonasal recess. Bulky medial and inferior rectus muscle of left eye with edematous palpabrae, along with proptosis of left eyeball with tenting of posterior aspect was seen suggesting features of Rhino Orbital Mucormycosis. Patient was started on treatment with Injection Amphotericin B, and was taken up for endoscopic debridement with orbital exentration following endoscopic Denker's approach.

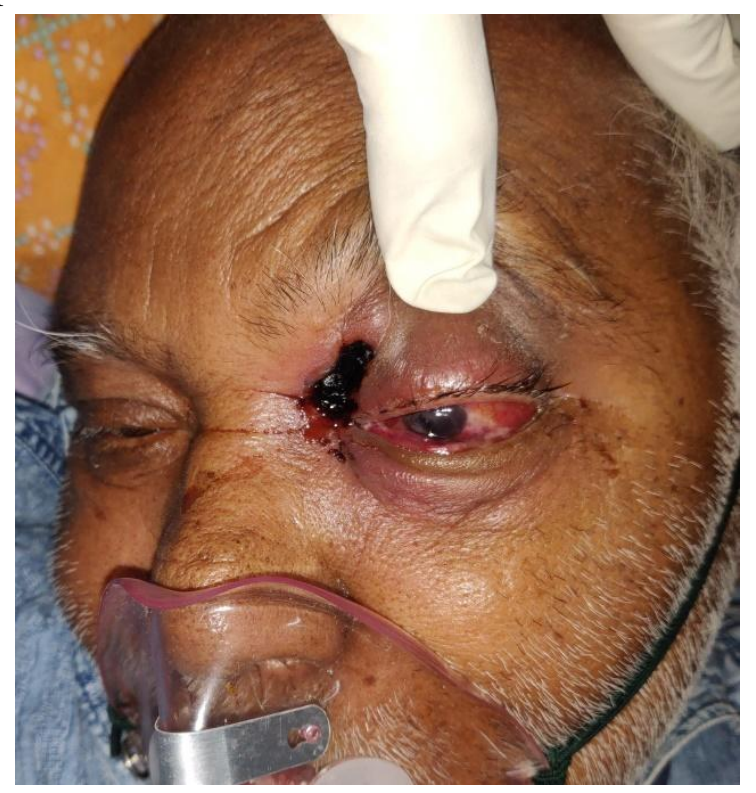

Case 3

82 year old male patient, k/c/o T2DM, hypertension, hyperthyroid and post TURP presented with pain and diminution of vision right eye, drooping of right upper lid, and right sided facial swelling. On examination, light perception and projection of rays was present. Patient had ptosis, proptosis, and periorbital edema of right eye. Mid dilated fixed pupil with absent light reflex was seen in right eye. Ocular motility was restricted in all directions of gaze. In left eye, visual acuity was PL+/PR+. Perorbital edema, conjunctival chemosis and congestion was seen in left eye with restricted ocular motility was present 
in all gaze directions. $\mathrm{KOH}$ mount of Deep Nasal swab showed aseptate broad based, ribbon like hyphae suggestive of mucor. Imaging with contrast suggested features of rhino orbital mucormycosis, and the patient was put on treatment accordingly.

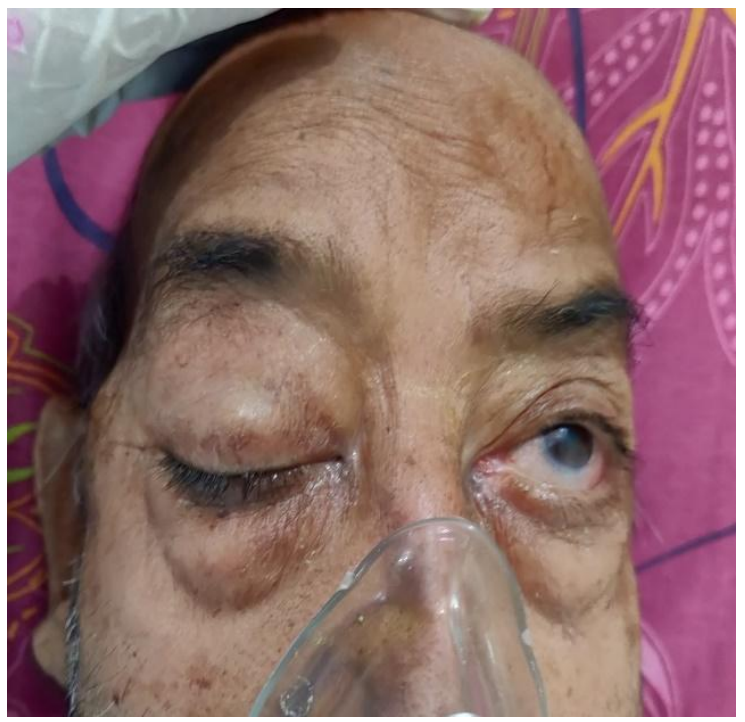

\section{Case 4}

58 years old diabetic male patient, known case of CKD k/c/o reactive arthritis, with severe anemia presented with sudden painful diminution of vision and swelling of right eye for two days. On examination of right eye, visual acuity was PL+, $\mathrm{PR}+$ in all quadrants. Patient had mild ptosis, proptosis, periorbital edema, conjunctival chemosis and sub conjunctival hemorrhage in right eye. Ocular motility was completely restricted in all positions of gaze. Diagnostic Nasal Endoscopy revealed black necrotic tissue in inferior turbinate, inferior meatus, middle septum upto skull base with right sided DNS. Histopathological examination and radiological imaging were consistent with mucormycosis. Patient was started on initial treatment with Amphotericin B following which Right maxillectomy was performed with Weber's Fergusson approach.

Demographic and clinical profile of patient along with major symptoms:

\begin{tabular}{|l|c|c|c|c|c|c|c|c|c|}
\hline S.no & Age/sex & $\begin{array}{c}\text { Eye } \\
\text { involved }\end{array}$ & $\begin{array}{c}\text { Visual } \\
\text { acuity }\end{array}$ & $\begin{array}{c}\text { Eye } \\
\text { ache }\end{array}$ & Headache & Ptosis & Proptosis & $\begin{array}{c}\text { Conjunctival } \\
\text { chemosis }\end{array}$ & Ophthalmoplegia \\
\hline 1. & $72 / \mathrm{F}$ & $\mathrm{Rt}$ & $6 / 12$ & + & + & + & + & - & + \\
\hline 2. & $55 / \mathrm{M}$ & $\mathrm{Lt}$ & $\mathrm{NPL}$ & + & - & + & + & + & + \\
\hline 3. & $58 / \mathrm{M}$ & $\mathrm{Rt}$ & PL+ & + & + & + & + & + & + \\
\hline 4. & $82 / \mathrm{M}$ & $\mathrm{Rt}$ & PL+ & + & + & + & + & - & + \\
\hline & & & & & & & & & \\
\hline & & & & & & & & & \\
\hline
\end{tabular}

$\mathrm{Rt}=$ Right, Lt=Left, NPL= No perception of light, $\mathrm{PL}=$ light perception present

\section{Discussion}

Mucormycosis is a condition with a fulminant course anda high mortality risk. The most common predisposing factoris DM (60-80\%), though hematologic diseases, neoplasias, chronic renal failure, antineoplastic agents, immunosuppressive therapy, corticosteroid use, protein-calorie malnutrition, organand bone marrow transplantation, and other conditions resulting in immunosuppression such as AIDS also factor in itsetiology.10 In a study by Yohai et al.7. including 145 cases, themost common predisposing factor was DM $(60 \%)$. Similarly, Ferry et al. ${ }^{(8)}$ found DM to be the greatest predisposing factorin their study population, with
83\%. Gumral et al. ${ }^{(9)}$ reviewed reports in the Turkish literature between 2000 and 2010 and found that among 79 cases of mucormycosis, the predisposing factor was diabetes in 32 cases and hematologic pathologies in 32 other cases. In our population, all 4 cases had diabetes mellitus.

With orbital involvement, clinical symptoms and findings include periorbitaledema, pain, proptosis, ophthalmoplegia and decreased vision. Yohai et al. ${ }^{(7)}$ reported periorbitaledema in $43 \%$ of their patients, periorbital pain in $11 \%$, proptosis in $64 \%$, ophthalmoplegia in $67 \%$ and vision impairment in $65 \%$. Among the 4 patients in our study, 3 had severe vision loss, while proptosis 
and restricted eye movement was observed in all four patients.

Amphotericin B has become the gold standard in the systemic treatment of mucormycosis. Liposomal amphotericin B is the first choice of treatment, as it crosses the blood-brain barrier more effectively. ${ }^{(11)}$

\section{References}

1. COVID-19 Treatment Guidelines Panel. Coronavirus disease 2019 (COVID-19) treatment guidelines. National Institutes of Health. 2020. [Last accessed on 2020 Dec 23].

2. Mehta S, Pandey A. Rhino-orbital mucormycosis associated with COVID-19. Cureus 2020;12:e10726.

3. Skiada A, Pavleas I, Drogari-Apiranthitou M. Epidemiology and diagnosis of mucormycosis: an update. J Fungi 2020;6:265.

4. Prakash H, Chakrabarti A. Global epidemiology of mucormycosis. J Fungi 2019;5:26.

5. Bavikar P., Mehta V. Rhino-orbitalcerebral mucormycosis: a fatal complication of uncontrolled diabetes mellitus. Cuneus. 2017;9:e1841. [PMC free article] [PubMed] [Google Scholar]

6. Afroze S.N., Korlepara R., Rao G.V., Madala J. Mucormycosis in a diabetic patient: a case report with an insight into its pathophysiology. Contemp. Clin. Dent. 2017;8:662-666. [PMC free article] [PubMed] [Google Scholar]

7. Yohai RA, Bullock JD, Aziz AA, Markert RJ. Survival factors in rhinoorbitalcerebral mucormycosis. Surv Ophthalmol. 1994;39:3-22.

8. Ferry AP, Abedi S. Diagnosis and management of rhino-orbito cerebral mucormycosis (Phycomycosis). A report of 16 personally observed cases. Ophthalmology. 1993;90:1096-1104.
9. Gumral R, Yildizoglu U, Saracli MA, Kaptan K, Tosun F, Yildiran ST. Acase of rhino orbital mucormycosis in a leukemic patient with a literature review from Turkey. Mycopathologia. 2011;172:397405.

10. Ferguson BJ. Mucormycosis of the nose and paranasal sinuses. Otolaryngol Clin North Am. 2000;33:349-365.

11. Petrikkos GL. Lipid formulations of amphotericin B as first-line treatment of zygomycosis. Clin Microbiol Infect. 2009; 15:87-92. 